\title{
Signatures of thermally excited vortices in a superconductor with competing orders
}

\author{
Gideon Wachtel and Dror Orgad \\ Racah Institute of Physics, The Hebrew University, Jerusalem 91904, Israel
}

(Dated: April 29, 2021)

\begin{abstract}
Experimental evidence for the existence of a fluctuating charge-density wave order in the pseudogap regime of $\mathrm{YBa}_{2} \mathrm{Cu}_{3} \mathrm{O}_{6+x}$ has renewed interest in its interplay with superconductivity. Here, we consider the problem within a nonlinear sigma model, which was recently proposed to describe the apparent competition between the two order parameters. In particular, we use a saddle-point approximation to calculate the properties of superconducting vortex excitations within such a model. In addition, we analytically calculate a collection of experimentally observable quantities, which probe both the superconducting and charge-density wave fluctuations, and identify expected signatures of thermally excited vortices.
\end{abstract}

\section{INTRODUCTION}

Over the past two decades, a large number of experiments have produced evidence that the pseudogap state of the cuprate high-temperature superconductors exhibits fluctuations towards various types of order. 1 In particular, recent X-ray scattering experiments ${ }^{2-12}$ indicate that the pseudogap regime of underdoped $\mathrm{YBa}_{2} \mathrm{Cu}_{3} \mathrm{O}_{6+x}$ is characterized by a local charge-density wave (CDW) order. The situation concerning the nature of superconducting (SC) fluctuations in the same compound is less clear. On the one hand, $c$-axis infrared spectroscopy ${ }^{13}$ has found signatures of a precursor SC state, which onsets close in temperature to the CDW signal. In addition, high-field torque magnetometry ${ }^{14}$ has detected fluctuation diamagnetism up to high temperatures. On the other hand, a different magnetometry measurement ${ }^{15}$ found diamagnetic response, consistent with Gaussian SC fluctuations, only in a narrow range above the critical temperature, $T_{c}$. The same conclusion was reached based on a measurement of the Nernst effect. 16

In any event, all available data point to the fact that the strength of the CDW fluctuations is anti-correlated with superconductivity. Specifically, the intensity of the CDW scattering peak grows as the system is cooled towards $T_{c}$, and then decreases upon entering the SC phase. Furthermore, the CDW signal is enhanced when a magnetic field is used to quench superconductivity. Finally, optical excitation of apical oxygen vibrations promotes transient superconducting signatures, $\stackrel{17,18}{=}$ resembling similar results in $\mathrm{La}_{2-x} \mathrm{Ba}_{x} \mathrm{CuO}_{4}$, where they were attributed to the melting of stripe order. Motivated by these findings Hayward et al ${ }^{19,20}$ have recently proposed a phenomenological non-linear sigma model (NLSM), which formulates the competition between fluctuating SC and CDW order parameters. Similar models emerge also from more microscopic considerations. ${ }^{21-23}$ Using MonteCarlo simulations of their model, Hayward et al. calculated the temperature dependence of the X-ray structure factor and of the diamagnetic susceptibility, and compared them to data from experiments on $\mathrm{YBa}_{2} \mathrm{Cu}_{3} \mathrm{O}_{6+x}$.

The purpose of the present paper is to analytically study the properties and expected experimental signatures of thermally excited SC vortices, within the NLSM of Ref. 19. In conventional BCS superconductors the core of the vortex consists of the normal metallic state. Consequently, the energy needed to create the core is of the order of the Fermi energy, thus making thermally excited vortices highly unlikely. However, this need not be the case when superconductivity competes with another state of comparable energy, as assumed in the NLSM. Such "cheap" vortices are required if one attempts to explain the diamagnetic and Nernst signals of underdoped cuprates within a vortex picture ${ }^{24,25}$. Experimentally, the checkerboard halos observed around vortices in a magnetic field ${ }^{26}$ give evidence that the vortex core may actually harbor local CDW order.

To make progress towards our goal we consider the NLSM in the limit of a large number, $N$, of fluctuating fields, and construct an effective theory for the SC vortices by integrating out the CDW degrees of freedom. Using the resulting theory we estimate the vortex core radius and core energy, from which we determine the density of thermally excited vortices. This allows us to identify the temperature range above the transition temperature over which vortices remain well defined and the physics is dominated by SC phase fluctuations. In this temperature range we calculate the magnetization, $M_{z}$, and the transverse thermoelectric transport coefficient, $\alpha_{y x}$, which is related to the Nernst signal. ${ }^{27}$ We find that both decay rapidly with temperature in a manner that is governed by the vortex core energy, while, on the other hand, the proliferation of vortices leads to a rise of the X-ray structure factor, $S_{C D W}$. As the temperature is increased further the system crosses over to a regime where the vortices are no longer well defined, amplitude fluctuations become important, and the fluctuations are nearly Gaussian. In this regime $M_{z}$ and $\alpha_{y x}$ continue to decrease, albeit in a more moderate fashion, and $S_{C D W}$ also becomes a decreasing function of temperature, thereby implying the existence of a peak.

The paper is organized as follows. In section II we present the model, consider its large- $N$ limit and identify the various temperature regimes which emerge. The derivation of an effective theory for the SC field is presented in section III which also contains a calculation of 
the vortex core energy and core size, as well as a numerical solution of the vortex structure. Section [IV discusses the expected diamagnetic and Nernst signals in different temperature regimes, while section $\nabla$ describes the maximum in the X-ray structure factor as a function of temperature. We conclude with a discussion of the relation to experiments in section VI

\section{THE MODEL AND ITS LARGE- $N$ EFFECTIVE THEORY}

Hayward et al $\underline{19}$ considered a real 6-dimensional order parameter, equivalent to a complex SC field $\Psi=n_{1}+i n_{2}$ and two complex CDW fields, $\Phi_{x}=n_{3}+i n_{4}$ and $\Phi_{y}=$ $n_{5}+i n_{6}$. In this paper we would like to use a saddle point approximation for the CDW fields, which is formally justified when their number is large. Thus, we analyze a system described by a complex SC field $\left\{\psi, \psi^{*}\right\}$, and $N-2$ real CDW fields $\left\{n_{\alpha}\right\}$, where $\alpha=1 \ldots N-2$. For the sake of simplicity we disregard quartic and anisotropic CDW terms, which appear in the Hamiltonian of Ref. 19, and analyze the more basic form

$$
H=\frac{\rho_{s}}{2} \int d^{2} r\left\{|\nabla \psi|^{2}+\sum_{\alpha=1}^{N-2}\left[\lambda\left(\nabla n_{\alpha}\right)^{2}+g n_{\alpha}^{2}\right]\right\} .
$$

Here $\rho_{s}$ is the stiffness of the SC order, $\lambda \rho_{s}$ is the corresponding quantity for the CDW components, and $g \rho_{s}$ is the energy density penalty for CDW ordering. Central to the model is the assumption that some type of order ( $\mathrm{SC}$ or CDW) is always locally present, in the sense of its amplitude, but that the different order parameters compete, as expressed by the constraint 28

$$
|\psi|^{2}+\sum_{\alpha=1}^{N-2} n_{\alpha}^{2}=N
$$

A free energy functional $F\left[\psi^{*}, \psi\right]$ for the $\mathrm{SC}$ field is obtained by integrating out the CDW fields

$$
\begin{aligned}
e^{-\beta F} & =\int \mathcal{D} n_{\alpha} \delta\left(|\psi|^{2}+\sum_{\alpha=1}^{N-2} n_{\alpha}^{2}-N\right) e^{-\beta H} \\
& =\int \mathcal{D} n_{\alpha} \mathcal{D} \bar{\sigma} e^{-\beta H+i \int d^{2} r \bar{\sigma}\left(|\psi|^{2}+\sum_{\alpha} n_{\alpha}^{2}-N\right)},
\end{aligned}
$$

where $\beta=1 / T$. In the limit $N \rightarrow \infty$ we carry out the integration over $n_{\alpha}$ while assuming that the Lagrange multiplier field $\bar{\sigma}$, which enforces the constraint, is fixed at its saddle point configuration $\bar{\sigma}=-i \sigma$. As a result

$$
\begin{aligned}
\beta F= & \frac{N-2}{2} \operatorname{Tr} \ln \left[\frac{1}{2} \beta \rho_{s}\left(-\lambda \nabla^{2}+g\right)+\sigma\right] \\
& +\int d^{2} r\left[\frac{1}{2} \beta \rho_{s}|\nabla \psi|^{2}+\sigma\left(|\psi|^{2}-N\right)\right],
\end{aligned}
$$

where $\sigma$ is determined by the saddle point equation

$$
\begin{aligned}
\frac{\delta \beta F}{\delta \sigma(\mathbf{r})}= & \frac{N-2}{2} \operatorname{Tr}\left[\left(\frac{1}{2} \beta \rho_{s}\left(-\lambda \nabla^{2}+g\right)+\sigma\right)^{-1} \delta_{\mathbf{r}}\right] \\
& +|\psi|^{2}-N=0,
\end{aligned}
$$

with $\delta_{\mathbf{r}}$ an operator, whose functional form is

$$
\delta_{\mathbf{r}}\left(\mathbf{r}^{\prime}, \mathbf{r}^{\prime \prime}\right)=\delta\left(\mathbf{r}^{\prime}-\mathbf{r}\right) \delta\left(\mathbf{r}^{\prime \prime}-\mathbf{r}\right) .
$$

The most likely SC configurations, those that minimize the free energy, are determined by the second saddle point equation

$$
\frac{\delta \beta F}{\delta \psi^{*}}=-\frac{1}{2} \beta \rho_{s} \nabla^{2} \psi+\sigma \psi=0 .
$$

Below the mean-field transition temperature, $T_{M F}$, Eqs. (517) acquire a uniform solution $\psi(\mathbf{r})=\psi_{0}, \sigma=0$ with

$$
\left|\psi_{0}\right|^{2}=N\left(1-\frac{T}{T_{M F}}\right)
$$

and

$$
\begin{aligned}
\frac{\rho_{s}}{T_{M F}} & =\frac{N-2}{N \lambda} \operatorname{Tr}\left[\left(-\nabla^{2}+g / \lambda\right)^{-1} \delta_{\mathbf{r}}\right] \\
& =\frac{N-2}{N \lambda} \int \frac{d^{2} p}{(2 \pi)^{2}} \frac{1}{p^{2}+g / \lambda} \\
& \simeq \frac{N-2}{4 \pi N \lambda}\left\{\begin{array}{cc}
\ln \left(32 \lambda / g a^{2}\right) & : \lambda / g a^{2} \gg 1 \\
4 \pi \lambda / g a^{2}+\mathcal{O}\left(\lambda^{2}\right) & : \lambda / g a^{2} \ll 1
\end{array}\right.
\end{aligned}
$$

Here, and wherever is needed in the following, we regularize the theory by putting it on a square lattice with lattice constant $a$. This amounts to replacing the Laplacian by its discrete version $p^{2} \rightarrow\left[4-2 \cos \left(p_{x} a\right)-2 \cos \left(p_{y} a\right)\right] / a^{2}$ and extending the momentum integration over the first Brillouin zone $\left|p_{x, y}\right|<\pi / a$.

Beyond the mean-field approximation $T_{M F}$ is only a crossover temperature, below which the most likely value of $\psi(\mathbf{r})$ assumes a finite amplitude. However, phase fluctuations, particularly in the form of vortices, prevent ordering down to a lower Berezinskii-KosterlitzThouless 29 temperature $T_{B K T}$. $T_{B K T}$ itself can be estimated using Monte-Carlo results $\frac{30}{}$ for the $X Y$-model on a square lattice, which when applied to our model gives $T_{B K T} \approx 0.9 \rho_{s}\left|\psi_{0}\right|^{2}$. Combined with Eq. (8) this implies $T_{M F} / T_{B K T}=1+T_{M F} / 0.9 \rho_{s} N$ and therefore the existence of a phase fluctuations regime, provided that our results hold down to $N=6$.

Therefore, it is possible to construct a schematic phase diagram, Fig. (1), in which we identify three temperature regions: (i) A high temperature regime, $T>T_{M F}$, approximately described by Gaussian fluctuations in both the SC and CDW fields; (ii) a superconducting phase fluctuations regime, $T_{B K T}<T<T_{M F}$, with thermally excited vortices; and, (iii) a SC phase for $T<T_{B K T}$. Eq. (9) indicates that for our case of interest, $\lambda / g a^{2}>1$, $T_{M F}$ grows approximately linearly with $\lambda$ and depends 


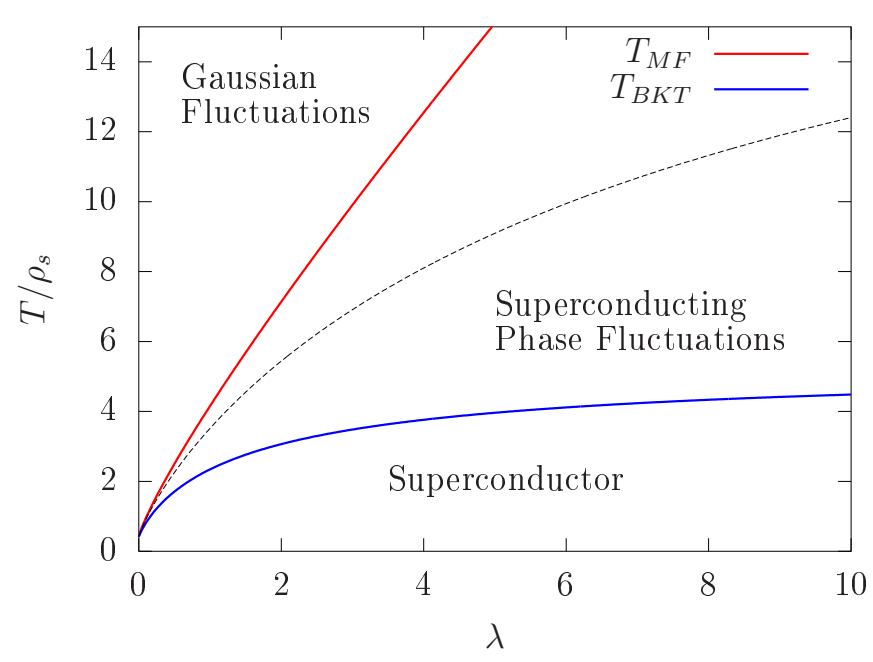

FIG. 1: Phase diagram as a function of $\lambda$ and temperature, $T$, obtained by extending our large $N$ results down to $N=6$. Here, $g a^{2}=0.3$. At low temperatures, $T<T_{B K T}$, the system is superconducting, while Gaussian fluctuations approximately describe the high temperatures regime, $T>T_{M F}$. Above $T_{B K T}$ the system enters a phase fluctuations regime, with well defined vortices up to an intermediate temperature, depicted by the dotted line. We define this curve as the temperature where $n_{f} r_{0}^{2} \approx 0.5$, and approximate it using Eqs. (27) and (31).

only weakly on $g$. Thus, the size of the phase fluctuations regime, (ii), is also expected to increase with $\lambda$. We note, however, that as the temperature is increased in this region, thermally excited vortices become denser and cease to be distinct objects. Consequently, significant amplitude fluctuations, associated with abundant vortex cores, are expected already at temperatures below $T_{M F}$. A more stringent definition of the phase fluctuations regime, would therefore require that the distance between thermally excited vortices be strictly larger than their size, i.e., $n_{f}<r_{0}^{-2}$. Even so, we still find that the extent of this regime grows with $\lambda$, as depicted by the dotted line in Fig. 1].

\section{THE VORTEX CORE}

\section{A. Effective Ginzburg-Landau theory and the vortex core size}

In the SC phase fluctuations regime, observables, such as the magnetization, $M_{z}$, and transverse thermoelectric transport coefficient, $\alpha_{y x}$, depend on the density, $n_{f}$, of thermally excited vortices. ${ }^{25,31-33}$ This density, in turn, is set by the energy, $\varepsilon_{c}$, and the linear size, $r_{0}$, of the vortex core $\frac{34}{4}$ Thus, in order to calculate observables in this temperature regime, one must first know the temperature dependence of the vortex core energy and size.

In order to estimate the vortex core size it is useful to construct a Ginzburg-Landau type theory for small values of $\psi$, which is applicable near the transition temperature $T_{M F}$, or, as in our case, at lower temperatures, but near the vortex center. When $\psi$ is small it is possible to write the free energy $F$ explicitly by expanding Eq. (4), and simultaneously solving the saddle point equation (5). For convenience, we define $\Sigma_{0}$ and $\Sigma_{1}$ by

$$
\frac{1}{2} \beta \rho_{s} \lambda\left(\Sigma_{0}+\Sigma_{1}\right)=\frac{1}{2} \beta \rho_{s} g+\sigma
$$

where, $\Sigma_{0} \sim \mathcal{O}(1)$ and $\Sigma_{1} \sim \mathcal{O}\left(|\psi|^{2} / N\right)$. We also introduce the operator $K$

$$
K=\left(-\nabla^{2}+\Sigma_{0}\right)^{-1}
$$

in terms of which the saddle point equation (5) takes the form

$$
\frac{N-2}{\beta \rho_{s} \lambda} \operatorname{Tr}\left[\left(K^{-1}+\Sigma_{1}\right)^{-1} \delta_{\mathbf{r}}\right]=N-|\psi|^{2} .
$$

$\Sigma_{0}$ itself is set by the zeroth order expansion of this equation in $|\psi|^{2} / N$,

$$
\frac{N-2}{\beta \rho_{s} \lambda} \operatorname{Tr}\left[K \delta_{\mathbf{r}}\right]=\frac{N-2}{\beta \rho_{s} \lambda} \int \frac{d^{2} p}{(2 \pi)^{2}} \frac{1}{p^{2}+\Sigma_{0}}=N .
$$

Regularizing the integral on the lattice we obtain for $T<$ $T_{M F}$

$$
\Sigma_{0}=\frac{32}{a^{2}} \exp \left(-\frac{4 \pi N \lambda}{N-2} \frac{\rho_{s}}{T}\right) .
$$

To first order in $|\psi|^{2} / N$, Eq. (12) implies the relation

$$
\frac{N-2}{\beta \rho_{s} \lambda} \operatorname{Tr}\left[K \Sigma_{1} K \delta_{\mathbf{r}}\right]=|\psi|^{2},
$$

whose Fourier transform reads

$$
\Sigma_{1}(\mathbf{q}) \int \frac{d^{2} p}{(2 \pi)^{2}} \frac{1}{p^{2}+\Sigma_{0}} \frac{1}{(\mathbf{p}+\mathbf{q})^{2}+\Sigma_{0}}=\frac{\beta \rho_{s} \lambda}{N-2}|\psi|^{2}(\mathbf{q}),
$$

leading for small $q$ to

$$
\Sigma_{1}=\frac{4 \pi \beta \rho_{s} \lambda}{N-2}\left(\Sigma_{0}-\frac{1}{6} \nabla^{2}\right)|\psi|^{2} .
$$

In terms of $\Sigma_{0}, \Sigma_{1}$ and $K$, the free energy, Eq. (44), is written as 


$$
\beta F=\frac{N-2}{2} \operatorname{Tr} \ln \left[\frac{1}{2} \beta \rho_{s} \lambda\left(K^{-1}+\Sigma_{1}\right)\right]+\frac{1}{2} \beta \rho_{s} \int d^{2} r\left[|\nabla \psi|^{2}+\left(\lambda \Sigma_{0}+\lambda \Sigma_{1}-g\right)\left(|\psi|^{2}-N\right)\right] .
$$

Expanding the trace in orders of $\Sigma_{1}$,

$$
\operatorname{Tr} \ln \left[\frac{1}{2} \beta \rho_{s} \lambda\left(K^{-1}+\Sigma_{1}\right)\right]=\operatorname{Tr} \ln \left[\frac{1}{2} \beta \rho_{s} \lambda K^{-1}\right]+\operatorname{Tr}\left[K \Sigma_{1}\right]-\frac{1}{2} \operatorname{Tr}\left[K \Sigma_{1} K \Sigma_{1}\right]+\cdots,
$$

and using Eqs. (13) and (17) we finally obtain a Ginzburg-Landau type free energy for $\psi$,

$$
F=\frac{1}{2} \rho_{s} \int d^{2} r\left[|\nabla \psi|^{2}+\left(\lambda \Sigma_{0}-g\right)|\psi|^{2}+\frac{2 \pi \beta \rho_{s} \lambda^{2}}{N-2} \Sigma_{0}|\psi|^{4}\right]
$$

where we have neglected gradients in the quartic term.

The theory described by Eq. (20) has a mean-field transition when $\lambda \Sigma_{0}-g=0$, which is consistent with Eq. (9). In addition, the linear size, $r_{0}$, of the vortex core in such a theory is roughly given by

$$
r_{0}^{-2} \sim-\left(\lambda \Sigma_{0}-g\right)
$$

According to Eq. (14), at low temperatures, $\Sigma_{0}$ is exponentially small, and $r_{0}^{-2} \sim g$ is independent of temperature.

\section{B. Condensation energy and vortex core energy}

The vortex core energy roughly scales as

$$
\varepsilon_{c} \sim U r_{0}^{2},
$$

where $U$ is the condensation energy density, i.e., the difference in free energy density between a state where SC is uniformly condensed, $\psi(\mathbf{r})=\psi_{0}$ and a state which is uniformly non-SC, $\psi(\mathbf{r})=0$. From Eq. (4) it follows that for the SC saddle point solution, $\psi(\mathbf{r})=\psi_{0}, \sigma=0$, the free energy density is

$$
\frac{F\left[\psi(\mathbf{r})=\psi_{0}\right]}{L^{2}}=\frac{(N-2) T}{2 L^{2}} \operatorname{Tr} \ln \left[-\lambda \nabla^{2}+g\right],
$$

with $L^{2}$ the system area. When $\psi(\mathbf{r})=0, \sigma$ assumes a different value, $\sigma=\beta \rho_{s}\left(\lambda \Sigma_{0}-g\right) / 2$, with $\Sigma_{0}$ given by Eq. (14). Substitution into Eq. (4) leads to

$$
\begin{aligned}
\frac{F[\psi(\mathbf{r})=0]}{L^{2}}= & \frac{(N-2) T}{2 L^{2}} \operatorname{Tr} \ln \left[-\lambda \nabla^{2}+\lambda \Sigma_{0}\right] \\
& -\frac{1}{2} \rho_{s}\left(\lambda \Sigma_{0}-g\right) N
\end{aligned}
$$

Combining the two results we find that the condensation energy density is given by

$$
\begin{aligned}
U= & \left(F[\psi(\mathbf{r})=0]-F\left[\psi(\mathbf{r})=\psi_{0}\right]\right) / L^{2} \\
= & \frac{(N-2) T}{2 L^{2}} \operatorname{Tr}\left[\ln \left(-\lambda \nabla^{2}+\lambda \Sigma_{0}\right)-\ln \left(-\lambda \nabla^{2}+g\right)\right] \\
& -\frac{1}{2} \rho_{s}\left(\lambda \Sigma_{0}-g\right) N .
\end{aligned}
$$

As noted above, $\Sigma_{0} \rightarrow 0$ at low temperatures. Therefore, in this limit $U$ behaves according to

$$
U \approx \frac{1}{2} \rho_{s} g N-\frac{(N-2) T}{2} \int \frac{d^{2} p}{(2 \pi)^{2}} \ln \left[\frac{p^{2}+g / \lambda}{p^{2}}\right] .
$$

In the same temperature regime, one finds from Eq. (21) that $r_{0}^{-2} \approx g$. Consequently, the main temperature dependence of $\varepsilon_{c}$ originates from $U$, which decreases linearly with $T$

$$
\frac{\varepsilon_{c}}{\rho_{s}} \sim \frac{N}{2}\left(1-b \frac{T}{\rho_{s}}\right)
$$

where

$$
b=\frac{N-2}{2 N} \int \frac{d^{2} p}{(2 \pi)^{2}} \ln \left[\frac{p^{2}+g / \lambda}{p^{2}}\right]=\frac{\rho_{s}}{T_{M F}}+\frac{N-2}{4 \pi N \lambda} .
$$

\section{Numerical solution of the vortex structure}

Although the above analytical estimate yields the general behavior of $\varepsilon_{c}$ as a function of $T$, it cannot give $\varepsilon_{c}$ in absolute values, since we do not know the correct proportionality constant which enters Eq. (22). In order to bridge this gap and check the validity range of the estimate, Eq. (27), we have calculated the vortex structure and energy numerically. The vortex configuration was obtained by solving the saddle point equations, (5) and (7), while imposing a phase winding $\psi(\mathbf{r})=f(r) e^{i \theta}$, where $\mathbf{r}$ is the position relative to the vortex center, $r=|\mathbf{r}|$, and $\theta$ is its angle with respect to the $x$ axis. For the solution we have used polar coordinates and discretized the radial coordinate $r$ in units of a short distance cutoff $a$. Figure 2 2 shows the amplitude $f(r)$ as a function of the distance from the vortex center, for a number of temperatures below $T_{M F}$. In the calculation we have set $N=6, g a^{2}=0.03$ and $\lambda=1$, which give $T_{M F} \approx 2.7 \rho_{s}$.

The vortex core energy was calculated by plugging the vortex solution into Eq. (44), subtracting from it the free 


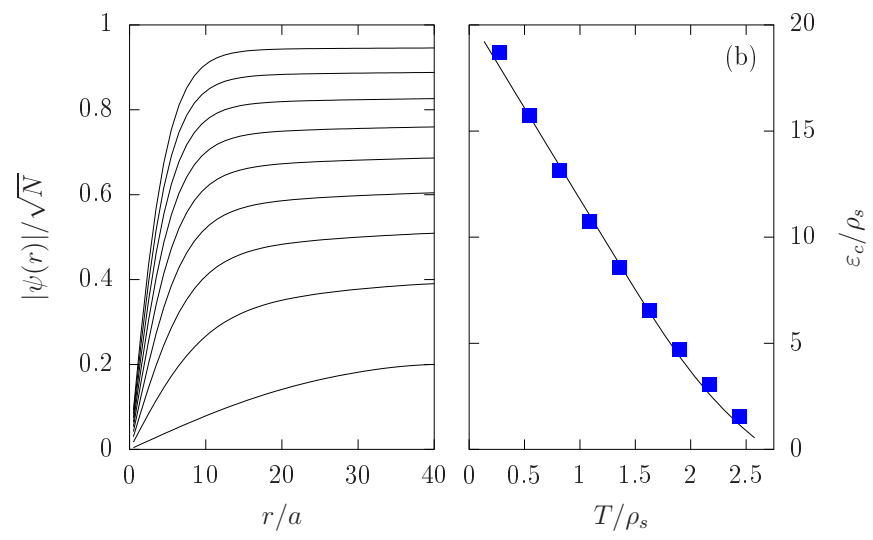

FIG. 2: (a) Amplitude of the SC field, $\psi$, as a function of the distance from a vortex core, for a number of temperatures below $T_{M F}$, obtained by numerically solving Eqs. (5) and (77) in polar coordinates. Here we take $N=6, \lambda=1$ and $g a^{2}=0.03$, where $a$ is a short distance cutoff. (b) Squares: vortex core energies, $\varepsilon_{c}$, for the vortices depicted in (a). Solid curve: the core energy calculated using Eqs. (21) and (25), where a numerical proportionality constant was selected, such that the curve agrees with the numerical solution.

energy of the uniform solution and the kinetic energy contribution $\rho_{s} / 2 \int d^{2} r f(r)^{2} / r^{2}$ of the superflow around the vortex core. The squares in Fig. $2 \mathrm{~b}$ depict the core energies for the vortex structures shown in Fig. 2ak, while the solid line gives the analytical $\varepsilon_{c}$ calculated on the lattice using Eqs. (21) and (25), multiplied by a constant in order to account for the unknown proportionality in Eq. (22). We have found an agreement between the analytical and numerical values for $\varepsilon_{c}$ over a wide range of parameters using a proportionality constant in the range $6.5-8$.

\section{DIAMAGNETISM AND NERNST EFFECT}

Knowing the structure of a vortex, its core energy, and size, it is now possible to calculate the temperature dependence of the magnetization, $M_{z}$, and transverse thermoelectric transport coefficient, $\alpha_{y x}$, in the phase fluctuations regime. It has been shown $25,31-33$, within a DebyeHückle theory for the thermally excited vortices, that

$$
M_{z}=-\frac{T B}{\phi_{0}^{2} n_{f}},
$$

where $\phi_{o}=h c / 2 e$ is the flux quantum, $B$ the magnetic field, and $n_{f}$ is the density of thermally excited vortices. Similarly, $\alpha_{y x}$, which relates the linear response of an electric current $J_{y}^{e}$ to a transverse thermal gradient $\partial_{x} T$ via $j_{y}^{e}=\alpha_{y x}\left(-\partial_{x} T\right)$, is given, in the Debye-Hückle regime, by ${ }^{25}$

$$
\alpha_{y x}=-\frac{c \varepsilon_{c} B}{\phi_{0}^{2} T n_{f}}=\frac{\varepsilon_{c}}{T} \frac{c M_{z}}{T} .
$$

The Debye-Hückle approximation is applicable in the range of temperatures above, but not to close to $T_{B K T}$, and at the same time low enough such that the distance between vortices is larger than their size. In this regime, and in the limit of small magnetic field, $B \rightarrow 0$, the density of thermally excited vortices is 34

$$
n_{f} \simeq 2 r_{0}^{-2} e^{-2 \varepsilon_{c} / T} .
$$

In principle, the $\varepsilon_{c}$ that one should use to determine $n_{f}$ is the renormalized core energy, which includes also the effect of fluctuations at short distances below the DebyeHückle screening length. However, outside the critical regime close to $T_{B K T}$ the renormalized $\varepsilon_{c}$ is roughly of the same order as its bare value. Since both $M_{z}$ and $\alpha_{y x}$ are inversely proportional to $n_{f}$, which exhibits an Arrhenius behavior, they both decay strongly with temperature. We take such a behavior as a signature of thermally excited vortices. It is important to note, though, that these features are not expected to appear so clearly in simulation because of finite size effects.

At high temperatures, $T>T_{M F}, M_{z}$ is approximately given by the $N \rightarrow \infty$ limit applied also to the SC fields $\frac{19}{1}$ It is also possible to carry out a similar $N \rightarrow \infty$ calculation $^{35}$ of $\alpha_{y x}$. The corresponding, high temperature results are given by

$$
M_{z}=-\frac{\pi T B}{3 \phi_{0}^{2} \Delta}
$$

and

$$
\alpha_{y x}=-\frac{\pi c B}{6 \phi_{0}^{2} \Delta}=\frac{c M_{z}}{2 T}
$$

where $\Delta$ is a solution to the following saddle-point equation

$$
\frac{N-2}{\beta \rho_{s}} \int \frac{d^{2} p}{(2 \pi)^{2}} \frac{1}{\lambda p^{2}+g+\Delta}+\frac{2}{\beta \rho_{s}} \int \frac{d^{2} p}{(2 \pi)^{2}} \frac{1}{p^{2}+\Delta}=N .
$$

Eqs. (32) and (33) are applicable as long as $\Delta a^{2}<1$. In this regime, and for the case $\lambda \geq 1$ and $g a^{2}<1$, Eq. (34) gives

$$
\Delta a^{2}=32\left(\frac{g a^{2}}{32 \lambda}\right)^{\frac{N-2}{N-2+2 \lambda} \frac{T_{M F}}{T}} .
$$

Therefore, we conclude that the rapid decay of both $M_{z}$ and $\alpha_{y x}$ in the phase fluctuations regime should crossover to a much slower decay as the temperature is increased through $T_{M F}$. Fig. 3 demonstrate this point by showing $-M_{z}$ and $-\alpha_{y x}$ for a square lattice with $\lambda=1$ and $g a^{2}=0.03$. For these parameters $T_{B K T} \approx 1.8 \rho_{s}$ and $T_{M F} \approx 2.7 \rho_{s}$. The phase fluctuations segment is based on Equations (29) and (30), while the high temperature segment is based on Equations (32) and (33). To determine $n_{f}$ we use in Eq. (31) the temperature dependent $\varepsilon_{c}$ as calculated in the previous section. We terminate the phase fluctuations segment when $n_{f} r_{0}^{2} \approx 0.35$, since 

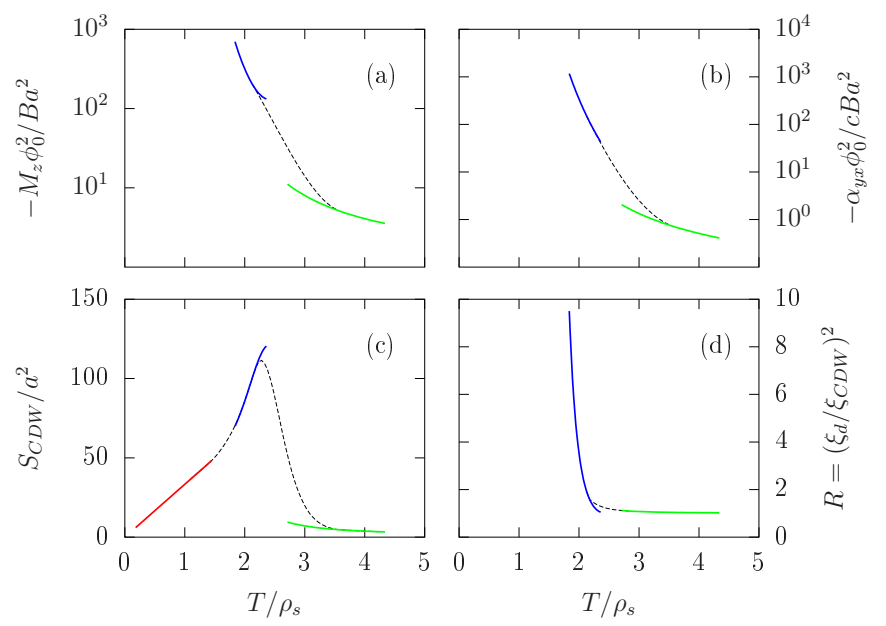

FIG. 3: (a) Magnetization, (b) transverse thermoelectric transport coefficient, (c) X-ray structure factor, and (d), the unitless ratio $R$, all as a function of temperature. Solid lines are based on analytic calculations, as explained in the main text, while the dashed lines are schematic interpolations in the crossover regions, intended as guides to the eye. These results were obtained for a square lattice of spacing $a, N=6$, $\lambda=1$ and $g a^{2}=0.03$.

at higher temperatures the vortices are no longer distinct objects, and the Debye-Hückle approximation is expected to fail. A schematic guide to the eye is depicted by the dashed curves, which interpolate the crossover between the phase fluctuations and Gaussian fluctuations regimes.

\section{X-RAY STRUCTURE FACTOR}

Recent X-ray scattering experiments show a pronounced maximum of the signal at certain incommensurate wave vectors, as a function of temperature. Hayward et al $\stackrel{19}{n}$ reproduced this maximum using Monte Carlo simulations of their NLSM. In addition, they were also able to demonstrate analytically that a maximum exits, by applying a $1 / N$ expansion to their model. Here we use our approach to show that the signal increases with temperature in a range of temperatures below $T_{M F}$, and decreases above. To do so, we calculate the CDW correlation function

$$
\begin{aligned}
G_{\alpha \beta}\left(\mathbf{r}, \mathbf{r}^{\prime}\right) & =\left\langle n_{\alpha}(\mathbf{r}) n_{\beta}\left(\mathbf{r}^{\prime}\right)\right\rangle \\
& =\frac{1}{Z} \int \mathcal{D} \psi \mathcal{D} \psi^{*} \mathcal{D} n_{\alpha} \mathcal{D} \bar{\sigma} n_{\alpha}(\mathbf{r}) n_{\beta}\left(\mathbf{r}^{\prime}\right) \\
& \times e^{-\beta H+i \int d^{2} r \bar{\sigma}\left(|\psi|^{2}+\sum_{\alpha} n_{\alpha}^{2}-N\right)},
\end{aligned}
$$

within our saddle-point approximation, where the integral over $\bar{\sigma}$ is replaced by its saddle-point value, given by Eq. (5), for each configuration of $\psi$.

Consider first low temperatures, $T<T_{B K T}$, where essentially there are no vortices. Ignoring SC amplitude fluctuations, which are expected to be small, we approx- imately have $\psi(\mathbf{r})=\psi_{0}$, which gives $\sigma=0$. Hence,

$$
\begin{aligned}
G_{\alpha \beta}(\mathbf{p}) & =\int d^{2} r e^{-i \mathbf{p} \cdot \mathbf{r}} G_{\alpha \beta}(\mathbf{r}, 0) \\
& \approx \frac{\delta_{\alpha \beta}}{\beta \rho_{s}\left(\lambda p^{2}+g\right)} .
\end{aligned}
$$

The X-ray structure factor, $S_{C D W}$, is obtained by taking the $\mathbf{p} \rightarrow 0$ limit of $G_{\alpha \beta}(\mathbf{p})$ with $\alpha=\beta$, thus,

$$
S_{C D W}\left(T<T_{B K T}\right) \approx \frac{T}{\rho_{s} g},
$$

which grows linearly with temperature.

At higher temperatures, thermally excited vortices appear in the system. In principle, one should average over such vortex configurations, each with its corresponding saddle-point solution, $\sigma$. Instead, we estimate their effect on $S_{C D W}$ by ignoring their spatial distribution and considering only their reduction of the average value of $|\psi|^{2}$. Specifically, we replace $|\psi|^{2}$ in Eq. (5), by its spatial average,

$$
\overline{|\psi|^{2}} \approx\left|\psi_{0}\right|^{2}-0.45 n_{f} r_{0}^{2},
$$

where the second term accounts for the vanishing $\psi$ inside the vortex cores. The numerical factor, 0.45 , was extracted from the numerical solutions of the vortex structure, which were described above in Section III. Since $S_{C D W}$ is a long-wavelength quantity we take the solution,

$$
\widetilde{\sigma}=\frac{g \rho_{s}}{2 T}\left[\left(\frac{g a^{2}}{32 \lambda}\right)^{\left(1-0.45 n_{f} r_{0}^{2}\right)\left(\frac{T_{M F}}{T}-1\right)}-1\right],
$$

of the resulting saddle-point equation as an approximation for all vortex configurations. This results in

$$
S_{C D W} \approx \frac{T}{\rho_{s} g+T \widetilde{\sigma}} .
$$

Since $\widetilde{\sigma}<0$, we find that $S_{C D W}$ curves upward for temperatures above $T_{B K T}$ but sufficiently below $T_{M F}$, such that vortices are distinct objects.

Above $T_{M F}$ the fluctuations become approximately Gaussian, and their effects can be obtained using the $N \rightarrow \infty$ limit also on the SC order parameter, as is described by Hayward et al..$^{19}$ Accordingly, the X-ray structure factor in the high temperature phase is given by

$$
S_{C D W}\left(T>T_{M F}\right) \approx \frac{T}{\rho_{s}(g+\Delta)} .
$$

where, as before, $\Delta$ is the solution of Eq. (34). The three solid segments in Figure $3 \mathrm{c}$ depict $S_{C D W}$ in the low temperature $\mathrm{SC}$ phase, the phase fluctuations regime, and the high temperature regime, as given respectively by Eqs. (38), (41) and (42). We therefore conclude that 
the maximum in the structure factor may be viewed to occur at the crossover from a phase fluctuations regime to a high temperature, Gaussian fluctuations regime.

From the correlation function, Eq. (36) it is also possible to extract the CDW correlation length, $\xi_{C D W}$. When $\sigma$ is approximately uniform, $G_{\alpha \beta}(\mathbf{p})$ has a Lorentzian form, whose width is defined to be $\xi_{C D W}^{-1}$, i.e., $G_{\alpha \beta}(\mathbf{p}) \sim$ $\left(p^{2}+\xi_{C D W}^{-2}\right)^{-1}$, or, $\xi_{C D W}^{2}=\lambda \rho_{s} S_{C D W} / T$. Hayward et $a l^{20}$ considered a dimensionless ratio between this correlation length and another length scale, $\xi_{d}$, which can be extracted from the diamagnetic magnetization by

$$
\xi_{d}^{2}=-\frac{3 \phi_{0}^{2} M_{z}}{\pi T B} .
$$

In the Gaussian limit, this is simply the SC correlation length while in the fluctuating vortices regime it roughly measures the distance between vortices. Using Monte Carlo simulations, Hayward et al. showed that the dimensionless ratio,

$$
R(T)=\left(\frac{\xi_{d}}{\xi_{C D W}}\right)^{2}
$$

decreases with temperature. Following our approach, we plot $R(T)$ in Figure $3 \mathrm{~d}$, in the phase fluctuations and high temperature regimes, with a schematic interpolation between them. At high temperatures described by Gaussian fluctuations one expects $R(T \rightarrow \infty)=1 / \lambda$. The experimental results for $R$ in Ref. 20 indicate that $R<1$ in this limit, therefore possibly implying $\lambda>1$.

\section{DISCUSSION}

The first question one must address when trying to apply our results to the $O(6)$ NLSM considered in Ref. 19, is whether the saddle-point approximation, appropriate when $N \rightarrow \infty$, is applicable to a model with a finite number of CDW fields. In the relevant model, there are four CDW fields, which do not, in two dimensions, order at any finite temperature. Without any CDW phases aside from a simple disordered phase, it is reasonable to expect that the saddle-point approximation captures the behavior of the CDW fields. Finite- $N$ corrections may, however, alter the numerical details of the solution, which can introduce discrepancies between the simulations and our results. On the other hand, our results would not be applicable in the presence of long range CDW order, which may, in principle, occur in layered systems with strong enough coupling between the layers.

As alluded to in the Introduction, the question of whether the cuprate high-temperature superconductors actually exhibit significant thermal fluctuations in the form of vortices, has been under debate. Here, we would like to ask what are the consequences of making such an assumption on the parameters that enter the NLSM. Consider first the range of temperatures above $T_{B K T}$ where one expects to find signatures of thermally excited vortices. Our results indicate that this range grows with $\lambda$. Experimentally, the large range of temperatures above $T_{c}$ in which there is a strong Nernst signal in a number of underdoped cuprates, has been advocated ${ }^{27}$ as evidence for the existence of thermally excited vortices in these systems. Quantitatively, it was claimed that phase fluctuations may exist up to at least $3 T_{c}$ in $\mathrm{La}_{2-x} \mathrm{Sr}_{x} \mathrm{CuO}_{4}$ and up to almost $2 T_{c}$ in $\mathrm{Bi}_{2} \mathrm{Sr}_{2} \mathrm{CaCu}_{2} \mathrm{O}_{8+\delta}$. This implies that one would need to take $\lambda>1$ in order to account for the large phase fluctuations regime. As we have noted above, experimental results for $R(T)$ may indicate that $\lambda>1$ in $\mathrm{YBa}_{2} \mathrm{Cu}_{3} \mathrm{O}_{6+x}$ as well. On the other hand, Hayward et al $\frac{19}{19}$ reproduced the X-ray structure factor maximum as a function of temperature using $\lambda=1$.

An additional point of comparison with experiments is the value of $\varepsilon_{c}$ at temperatures just above $T_{B K T}$. For $\lambda=$ 1 and $g a^{2}=0.3$ we find the ratio $\varepsilon_{c}\left(T \gtrsim T_{B K T}\right) / T_{B K T} \approx$ 3 , which increases for larger $\lambda$. Analyzing Nernst measurements from the point of view of vortex fluctuations, we have estimated 25 that in $\mathrm{La}_{2-x} \mathrm{Sr}_{x} \mathrm{CuO}_{4} \varepsilon_{c} / T_{c} \approx 4-5$, which is consistent with $\lambda>1$. Within the context of our calculation it seems that $\varepsilon_{c} / T_{c}$ cannot be any larger in the NLSM. However, an analysis 36 of finite-frequency sheet conductivity in underdoped $\mathrm{Y}_{1-x} \mathrm{Ca}_{x} \mathrm{Ba}_{2} \mathrm{Cu}_{3} \mathrm{O}_{7-\delta}$ films has concluded that $\varepsilon_{c} / T_{c} \approx 8$. In any event, it seems that $\varepsilon_{c}$ is much smaller than its BCS value, which is of the order of the Fermi energy. By construction, the NLSM contains this piece of phenomenology, as its energetics is set by $\rho_{s}$. However, to understand this fact one must consider the microscopic details at the basis of the phenomenological model.

\section{Acknowledgments}

This research was supported by the Israel Science Foundation (Grant No. 585/13).
${ }^{1}$ For a recent review see, E. Fradkin, S. A. Kivelson, and J. M. Tranquada, arXiv:1407:4480 (2014).

2 G. Ghiringhelli, M. Le Tacon, M. Minola, S. BlancoCanosa, C. Mazzoli, N. B. Brookes, G. M. De Luca, A. Frano, D. G. Hawthorn, F. He, T. Loew, M. Moretti Sala, D. C. Peets, M. Salluzzo, E. Schierle,
R. Sutarto, G. A. Sawatzky, E. Weschke, B. Keimer, and L. Braicovich, Science 337, 821 (2012).

3 J. Chang, E. Blackburn, A. T. Holmes, N. B. Christensen, J. Larsen, J. Mesot, R. Liang, D. A. Bonn, W. N. Hardy, A. Watenphul, M. v. Zimmermann, E. M. Forgan, and S. M. Hayden, Nature Phys. 8, 871 (2012). 
4 A. J. Achkar, R. Sutarto, X. Mao, F. He, A. Frano, S. Blanco-Canosa, M. Le Tacon, G. Ghiringhelli, L. Braicovich, M. Minola, M. Moretti Sala, C. Mazzoli, R. Liang, D. A. Bonn, W. N. Hardy, B. Keimer, G. A. Sawatzky, and D. G. Hawthorn, Phys. Rev. Lett. 109, 167001 (2012).

${ }^{5}$ E. Blackburn, J. Chang, M. Hücker, A. T. Holmes, N. B. Christensen, R. Liang, D. A. Bonn, W. N. Hardy, U. Rütt, O. Gutowski, M. v. Zimmermann, E. M. Forgan, and S. M. Hayden, Phys. Rev. Lett. 110, 137004 (2013).

6 A. J. Achkar, X. Mao, C. McMahon, R. Sutarto, F. He, R. Liang, D. A. Bonn, W. N. Hardy, and D. G. Hawthorn, Phys. Rev. Lett. 113, 107002 (2014).

7 R. Comin, A. Frano, M. M. Yee, Y. Yoshida, H. Eisaki, E. Schierle, E. Weschke, R. Sutarto, F. He, A. Soumyanarayanan, Yang He, M. Le Tacon, I. S. Elfimov, J. E. Hoffman, G. A. Sawatzky, B. Keimer, and A. Damascelli, Science 343, 390 (2014).

8 E. H. da Silva Neto, P. Aynajian, A. Frano, R.Comin, E. Schierle, E. Weschke, A. Gyenis, J. Wen, J. Schneeloch, Z.Xu, S. Ono, G. Gu, M. Le Tacon, and A. Yazdani, Science 343, 393 (2014).

9 M. Le Tacon, A. Bosak, S. M. Souliou, G. Dellea, T. Loew, R. Heid, K.-P. Bohnen, G. Ghiringhelli, M. Krisch, and B. Keimer, Nat. Phys. 10, 52 (2014).

10 R. Comin, R. Sutarto, F. He, E. da Silva Neto, L. Chauviere, A. Frano, R. Liang, W. N. Hardy, D. Bonn, Y. Yoshida, H. Eisaki, J. E. Hoffman, B. Keimer, G. A. Sawatzky, and A. Damascelli, arXiv:1402.5415 (2014).

11 M. Hücker, N. B. Christensen, A. T. Holmes, E. Blackburn, E. M. Forgan, R. Liang, D. A. Bonn, W. N. Hardy, O. Gutowski, M. v. Zimmermann, S.M. Hayden, and J. Chang, arXiv:1405.7001 (2014).

12 S. Blanco-Canosa, A. Frano, E. Schierle, J. Porras, T. Loew, M. Minola, M. Bluschke, E. Weschke, B. Keimer, and M. L. Tacon, arXiv:1406.1595 (2014).

13 A. Dubroka, M. Rössle, K. W. Kim, V. K. Malik, D. Munzar, D. N. Basov, A. A. Schafgans, S. J. Moon, C. T. Lin, D. Haug, V. Hinkov, B. Keimer, Th. Wolf, J. G.Storey, J. L. Tallon, and C. Bernhard, Phys. Rev. Lett. 106, 047006 (2011).

${ }^{14}$ F. Yu, M. Hirschberger, T. Loew, G. Li, B. J. Lawson, T. Asaba, J. B. Kemper, T. Liang, J. Porras, G. S. Boebinger, J. Singleton, B. Keimer, L. Li, and N. P. Ong, arXiv:1402.7371 (2014).

15 I. Kokanović, D. J. Hills, M. L. Sutherland, R. Liang, and J. R. Cooper, Phys. Rev. B 88, 060505(R) (2013).

${ }^{16}$ R. Daou, J. Chang, D. LeBoeuf, O. Cyr-Choinière,
F. Lalibertè, N. Doiron-Leyraud, B. J. Ramshaw, R. Liang, D. A. Bonn, W. N. Hardy, and L. Taillefer, Nature (London) 463, 519 (2010).

17 W. Hu, S. Kaiser, D. Nicoletti, C. R. Hunt, I. Gierz, M. C. Hoffmann, M. Le Tacon, T. Loew, B. Keimer, and A. Cavalleri, Nat. Mater. 13, 705 (2014).

18 S. Kaiser, C. R. Hunt, D. Nicoletti, W. Hu, I. Gierz, H. Y. Liu, M. Le Tacon, T. Loew, D. Haug, B. Keimer, and A. Cavalleri, Phys. Rev. B 89, 184516 (2014).

19 L. E. Hayward, D. G. Hawthorn, R. G. Melko, and S. Sachdev, Science 343, 1336 (2014).

20 L. E. Hayward, A. J. Achkar, D. G. Hawthorn, R. G. Melko, and S. Sachdev, Phys. Rev. B 90, 094515 (2014).

21 M. A. Metlitski and S. Sachdev, Phys. Rev. B 82, 075128 (2010).

22 K. B. Efetov, H. Meier, and C. Pépin, Nature Phys. 9, 442 (2013).

23 H. Meier, M. Einenkel, C. Pépin, and K. B. Efetov, Phys. Rev. B 88, 020506(R) (2013).

24 P. A. Lee, N. Nagaosa, and X. Wen, Rev. Mod. Phys. 78, 17 (2006)

25 G. Wachtel and D. Orgad, arXiv:1310.2993 (2013).

26 J. E. Hoffman, E. W. Hudson, K. M. Lang, V. Madhavan, H. Eisaki, S. Uchida, and J. C. Davis, Science 295, 466 (2002).

27 Y. Wang, L. Li, and N. P. Ong, Phys. Rev. B 73, 024510 (2006).

28 We choose to normalized the fields to $N$, which should be compared with the convention used by Hayward et al. ${ }^{19}$, who normalize the fields to 1 . As a result, when comparing our results with their's, one needs to rescale $\rho_{s}$ by $N$.

29 J. M. Kosterlitz and D. J. Thouless, J. Phys. C 6, 1181 (1973).

30 P. Ollson and P. Minnhagen, Phys. Scr. 43, 203 (1991); N. Schultka and E. Manousakis, Phys. Rev. B 49, 12071 (1994).

31 B. I. Halperin and D. R. Nelson, J. Low Temp. Phys. 36, 599 (1979).

${ }^{32}$ V. Oganesyan, D. A. Huse, and S. L. Sondhi, Phys. Rev. B 73, 094503 (2006).

33 L. Benfatto, C. Castellani, and T. Giamarchi, Phys. Rev. Lett. 99, 207002 (2007).

34 P. Minnhagen, Rev. Mod. Phys. 59, 1001 (1987).

35 G. Wachtel and D. Orgad, arXiv:1410.0159 (2014).

36 L. Benfatto, C. Castellani, and T. Giamarchi, Phys. Rev. B 77, 100506(R) (2008). 